\title{
Irrational Versus Rational Charge and Statistics in Two-Dimensional Quantum Systems
}

\author{
Claudio Chamon, ${ }^{1,2}$ Chang-Yu Hou, ${ }^{1}$ Roman Jackiw, ${ }^{3}$ Christopher Mudry, ${ }^{4}$ So-Young Pi, ${ }^{1}$ and Andreas P. Schnyder ${ }^{5}$ \\ ${ }^{1}$ Physics Department, Boston University, Boston, Massachusetts 02215, USA \\ ${ }^{2}$ Department of Physics, Massachusetts Institute of Technology, Cambridge, Massachusetts 02139, USA \\ ${ }^{3}$ Department of Physics, Massachusetts Institute of Technology, Cambridge, Massachusetts 02139, USA \\ ${ }^{4}$ Condensed matter theory group, Paul Scherrer Institut, CH-5232 Villigen PSI, Switzerland \\ ${ }^{5}$ Department of Physics, University of California, Santa Barbara, California 93106, USA
}

(Received 3 July 2007; revised manuscript received 14 November 2007; published 21 March 2008)

\begin{abstract}
We show that quasiparticle excitations with irrational charge and irrational exchange statistics exist in tight-binding systems described, in the continuum approximation, by the Dirac equation in $(2+$ 1)-dimensional space and time. These excitations can be deconfined at zero temperature, but when they are, the charge rerationalizes to the value $1 / 2$ and the exchange statistics to that of "quartons" (halfsemions).
\end{abstract}

DOI: 10.1103/PhysRevLett.100.110405

Introduction. - It was shown by Jackiw and Rebbi [1] and by Su, Schrieffer, and Heeger [2] that excitations with fermion number $1 / 2$ (or charge $1 / 2$ ) exist at domain walls in the dimerization pattern of electrons hopping along a chain, as is believed to occur in polyacetylene. For electrons hopping on the honeycomb lattice, the lattice relevant to graphene, a topological defect in a dimerization pattern that is realized by a vortex was shown to lead to a topological zero mode and bind the fermion number $1 / 2$ to the vortex [3]. Fermion-number fractionalization in both polyacetylene and graphene can be understood in terms of the spectral properties of one-dimensional (1D) and twodimensional (2D) massive Dirac Hamiltonians, respectively, that describe the low energy limit of the electronic tight-binding Hamiltonians. These fractionally charged topological excitations are generically deconfined in 1D. Their deconfinement in 2D relies on a mechanism for the screening of the 2D Coulomb potential by thermal [3] or quantum fluctuations involving an axial gauge field [4].

Applying different potentials to odd and even sites of the linear chain results in a continuously varying fractional fermion number [5-7]. At the level of the Dirac Hamiltonian, this perturbation is represented by a second (gap-opening) mass term, that adds in quadrature to the mass due to the hopping dimerization of the chain. A complex order parameter is constructed from these two masses as its real and imaginary pieces, and the fractional fermion number is related to the phase twist of this order parameter as it sweeps through a domain wall, a result that has a natural interpretation within a bosonization scheme [5]. Although exchange statistics is ill-defined in 1D, this varying phase twist also implies a continuously varying exclusion statistics [8].

Can the charge and the exchange statistics of fractionalized quasiparticles in 2D be continuously varied as well? Here we show that they can. The fermion numbers of quasiparticles bound to a vortex can thus be irrational. Remarkably, if an axial gauge field supporting a halfvortex is added to (precisely) screen the interaction poten-
PACS numbers: 05.30.Pr

tial between quasiparticles, their fractional fermion number rerationalizes to the value $Q=1 / 2$ and their statistical angle (for when time-reversal symmetry is broken) to the value $\theta / \pi=1 / 4$. These results are first derived at the level of an effective field theory in $(2+1)$-dimensional space and time. We then discuss the relevance of this analysis for planar tight-binding models.

Definitions. - The massive Dirac Lagrangian that we shall consider in this Letter takes the form

$$
\mathcal{L}:=\bar{\Psi}\left[\gamma^{\mu}\left(i \partial_{\mu}+\gamma_{5} A_{5 \mu}\right)-M_{a} \Delta_{a}\right] \Psi,
$$

with $\mu=0,1,2$ and $a=0,1,2,3, \bar{\Psi} \equiv \Psi^{\dagger} \gamma^{0}$, and $4 \times 4$ matrices

$$
\begin{array}{cc}
\gamma^{0} \equiv\left(\begin{array}{cc}
0 & I \\
I & 0
\end{array}\right), & \gamma^{i} \equiv\left(\begin{array}{cc}
0 & -\sigma_{i} \\
\sigma_{i} & 0
\end{array}\right), \\
\gamma_{5} \equiv i \gamma^{0} \gamma^{1} \gamma^{2} \gamma^{3}, & M_{1}=\mathbb{1}, \quad M_{2}=-i \gamma_{5}, \\
M_{3}=\gamma^{3}, & M_{0}=\gamma_{5} \gamma^{3} .
\end{array}
$$

We allow for the background fields $A_{5 \mu}$ and $\Delta_{a}$ to vary in space-time $x \equiv\left(x^{\mu}\right) \equiv(t, \boldsymbol{r})$. The field $A_{5 \mu}$ couples to the Dirac fermions as an axial $U(1)$ gauge field does [4]. The four fields $\Delta_{a}$, when constant in space and time, open an energy gap in the Dirac spectrum. These masses have the following physical meaning on the honeycomb lattice, e.g., graphene. The masses $\Delta_{1,2}$ correspond to the two components of the complex Kekule bond-density-wave order parameter $\Delta \equiv \Delta_{1}+i \Delta_{2}$ [3]. The mass $\Delta_{3} \equiv \mu_{s}$ is a staggered chemical potential that favors charges to sit in one of the sublattices of the honeycomb lattice [9]. Finally, $\Delta_{0}=\eta$ is the only of the four masses that breaks timereversal symmetry (TRS). It originates on the lattice from a next-neighbor hopping term with phases that was introduced in Ref. [10]. We will use a constant $\eta$ to discuss quasiparticle statistics. The TRS masses $\mu_{s}, \Delta_{1}$, and $\Delta_{2}$ add in quadrature. Hence, we define the fields $m$ and $\boldsymbol{n}=$ $\left(n_{1}, n_{2}, n_{3}\right)$ by

$$
m:=\sqrt{\Delta_{1}^{2}+\Delta_{2}^{2}+\mu_{\mathrm{s}}^{2}}, \quad n_{i}:=\frac{\Delta_{i}}{m} .
$$


Induced fermionic $U(1)$-charge current with TRS. - The current $j^{\mu}(x) \equiv\left\langle\bar{\Psi}(x) \gamma^{\mu} \Psi(x)\right\rangle$ can be computed perturbatively in a derivative expansion in $\boldsymbol{n}$. When $A_{5}^{\mu}=0$, it reads

$$
j^{\mu}=\frac{1}{8 \pi} \epsilon^{\mu \nu \rho} n \cdot\left(\partial_{\nu} n \wedge \partial_{\rho} n\right) .
$$

Equation (3) is the desired extension to 2D of the bosonized current in a Luttinger liquid. For a static charge-1 vortex that vanishes at the origin and has the large distance asymptotic form

$$
\begin{aligned}
\Delta(\boldsymbol{r}) & =\Delta(\infty) e^{i \phi}+\mathcal{O}\left(r^{-2}\right), \\
\partial_{i} \Delta(\boldsymbol{r}) & =-i \Delta(\infty) \frac{\epsilon_{i j} r^{j}}{r} e^{i \phi}+\mathcal{O}\left(r^{-3}\right),
\end{aligned}
$$

where $r=r(\cos \phi$, sin $\phi)$, we find the charge

$$
Q=\frac{1}{2}\left(1-\frac{\mu_{\mathrm{s}}(\infty) / \Delta(\infty)}{\sqrt{1+\mu_{\mathrm{s}}^{2}(\infty) / \Delta^{2}(\infty)}}\right) \quad(\bmod 1)
$$

that varies continuously with the asymptotic value of the dimensionless ratio $\mu_{s}(\infty) / \Delta(\infty)$. The ambiguity mod 1 in the fermion number $Q$ corresponds to whether the bound state in the gap is empty or occupied. For $\mu_{s}(\infty)=0, Q=$ $\pm 1 / 2$ is recovered as in Ref. [3].

When $A_{5}^{\mu} \neq 0$, the induced fermionic current is $J^{\mu}=$ $j_{\mathrm{cov}}^{\mu}+\delta j^{\mu}$ where $j_{\mathrm{cov}}^{\mu}$ is Eq. (3) in which the derivatives have been replaced by the covariant derivatives $D^{\mu}=$ $\partial^{\mu}+2 i A_{5}^{\mu}, \quad \delta j^{\mu}=-\frac{1}{2 \pi} \frac{\mu_{\mathrm{s}}}{m} F_{5}^{\mu}$, and $F_{5}^{\mu}=e^{\mu \nu \rho} \partial_{\nu} A_{5 \rho}$. The current $J^{\mu}$ is conserved. The additional charge contributed by $\delta j^{\mu}$ is proportional to the flux carried by $A_{5 \mu}$. In fact, in the presence of the static vortex (4) and of the static half-vortex $A_{5}^{i}(\boldsymbol{r})=-\epsilon^{i j} \frac{r^{j}}{r^{2}} a_{5}(r)$ where $a_{5}(0)=0$ and $a_{5}(\infty)=1 / 2$, the fractional charge is $Q=1 / 2$ for any $\mu_{\mathrm{s}}(\infty) / \Delta(\infty)$ !

Observe that, when $A_{5 \mu}=0$, despite the fact that the Dirac Hamiltonian associated with Eq. (1) does not possess an $S U(2)$ symmetry [the mass matrices $M_{1,2,3}$ do not close an $S U(2)$ algebra under multiplication], the induced fermionic current (3) is the same as that in the $O(3)$ nonlinear sigma model (NLSM) derived from Dirac Hamiltonians with an internal $S U(2)$ symmetry $[11,12]$. This is so for the following reason. The nonunitary transformation $\bar{\Psi}=$ $\bar{\chi} M_{0}$ and $\Psi=\chi$ is harmless as it induces a constant Jacobian that cancels for any physical observable. Under this transformation, the matrices $\Sigma_{i} \equiv M_{0} M_{i}$ close the $S U(2)$ algebra $\left[\Sigma_{i}, \Sigma_{j}\right]=2 i \epsilon_{i j k} \Sigma_{k}$ and commute with the transformed matrices $\Gamma^{\mu}=M_{0} \gamma^{\mu}$. The transformed Lagrangian (1) is then invariant under the $S U(2)$ transformations generated by $\mathbf{\Sigma}=\left(\Sigma_{1}, \Sigma_{2}, \Sigma_{3}\right)$ when $A_{5}^{\mu}=0$.

Exchange statistics. - If two identical quasiparticles associated with two vortices are exchanged, the manyparticle wave function changes by the phase $\exp (i \theta)$ with $\theta$ the statistical angle. On general grounds, we expect that fractionally charged quasiparticles acquire a mutual fractional statistics. However, for quasiparticles to display fractional statistics one needs to break TRS, and thus we now include a uniform TRS-breaking $\Delta_{0} \equiv \eta$. The calculation of the fractional charge when $\eta=0$ has shown that fractionalization is of topological origin in that it only depends on the asymptotic values of the fields. The same fractional charge follows when we impose the nonlinear constraint $\boldsymbol{n}^{2}=n_{1}^{2}+n_{2}^{2}+n_{3}^{2}=1$, with $m$ in Eq. (2) held constant (see Fig. 1). Now, a uniform $\eta$ competes with a uniform $m$ leading to a quantum critical point when $m=$ $\eta$. Correspondingly, the fractional charge can be computed for a nonvanishing ratio $\eta / m$ and shown to be the same as before except for the multiplicative factor $\kappa_{C}:=\Theta(m-$ $|\eta|)$ with $\Theta$ the Heaviside step function. Moreover, for $A_{5}^{\mu}=0$, the effective action governing the dynamics of the background fields $\boldsymbol{n}$ is, despite the lack of $S U(2)$ symmetry in Eq. (1), the same as that of the $O(3)$ NLSM of Refs. [11,12]:

$$
S_{\text {eff }}=\frac{m}{8 \pi} \int d^{3} x\left(\partial_{\mu} \boldsymbol{n}\right)^{2}+i \pi \kappa_{\mathrm{H}} \operatorname{sgn}(\eta) S_{\mathrm{Hopf}}+\ldots
$$

The Hopf term is nonlocal in terms of the fields $\mathbf{n}$. One possible representation is [13] (see also Ref. [14])

$$
S_{\text {Hopf }}=\frac{\epsilon_{\mu \nu \rho}}{48 \pi^{2}} \int d^{3} x \operatorname{tr}\left[\left(U i \partial^{\mu} U^{\dagger}\right)\left(U i \partial^{\nu} U^{\dagger}\right)\left(U i \partial^{\rho} U^{\dagger}\right)\right]
$$

with $U$ the unitary $4 \times 4$ matrix field that rotates the spacetime dependent vector $\boldsymbol{n}$ into a fixed direction, say $(0,0,1)$ : $\boldsymbol{\Sigma} \cdot \boldsymbol{n}=U^{\dagger} \Sigma_{3} U$. When the angle $\kappa_{\mathrm{H}}:=\Theta(|\eta|-m)$ is nonvanishing, the angle $\kappa_{\mathrm{C}}$ vanishes and vice versa. Both the fractional charge and the Hopf term contribute to the statistical angle,

$$
\theta / \pi=\operatorname{sgn}(\eta) Q^{2}\left(\kappa_{\mathrm{C}}+\kappa_{\mathrm{H}}\right)=\operatorname{sgn}(\eta) Q^{2} .
$$

A heuristic derivation of Eq. (7) when $m>|\eta|$ and $A_{5}^{\mu}=0$ is the following. Consider first a uniform $\boldsymbol{n}$, for which the square of the Dirac Hamiltonian $\mathcal{H}$ associated with Eq. (1) is $\mathcal{H}^{2}=\left(\boldsymbol{p}^{2}+m^{2}+\eta^{2}\right)+2 \eta \sum_{i=1}^{3} \Sigma_{i} n_{i}$. This Hamiltonian resembles that of a particle with spin in a magnetic field, of strength proportional to $\eta$, that points in the $\boldsymbol{n}$ direction (hereafter, we take $\eta>0$ ). If the uniform $\boldsymbol{n}$ is rotated adiabatically by $2 \pi$ around the $\hat{z}$ direction, a

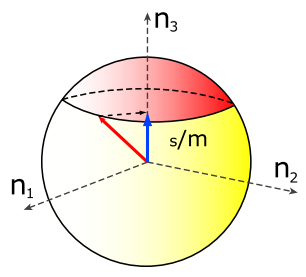

FIG. 1 (color online). The 2 sphere spanned by the masses $\boldsymbol{n} \equiv$ $\left(n_{1}, n_{2}, n_{3}\right)=m^{-1}\left(\Delta_{1}, \Delta_{2}, \mu_{s}\right)$. A vortex of charge 1 in the dimerizations $\Delta_{1}$ and $\Delta_{2}$ corresponds to a parallel on the sphere whose height is fixed by the asymptotic value of the staggered chemical potential $\mu_{s}(\infty)$. The spherical area red (or yellow) enclosed by this parallel gives the fractional charge induced by the fermionic zero mode localized at the core of the vortex. 
Berry phase $\gamma_{\text {rot }}(\boldsymbol{n})=2 \pi \sin ^{2} \frac{\alpha}{2}$ is accumulated for each filled single-particle electronic state, where $\alpha$ is the angle that $\boldsymbol{n}$ makes with the north pole of the unit sphere. The many-body Berry phase is the sum over occupied singleparticle Berry phases after subtraction of the Berry phase of some reference many-body state (i.e., without vortex). Now, $\boldsymbol{n}$ is not spatially uniform for a vortex, and thus if all $\boldsymbol{n}(\boldsymbol{r})$ are rigidly rotated by $2 \pi$, their accumulated phase depends on the texture. In a semiclassical approximation, the Berry phase at each $\boldsymbol{r}$ must be weighted by the fermion density: $\gamma_{\text {rot }}=\int d^{2} \boldsymbol{r} j^{0}(\boldsymbol{r}) 2 \pi \sin ^{2} \frac{\alpha(\boldsymbol{r})}{2}$. With the help of Eq. (3) when $m>\eta$, this gives $\gamma_{\text {rot }}=\int \frac{d \Omega}{4 \pi} 2 \pi \sin ^{2} \frac{\alpha}{2}=$ $\pi \sin ^{4} \frac{\alpha}{2}=\pi Q^{2}$. Hence, the phase accumulated by spinning the vortex by $2 \pi$ is $\pi Q^{2}$, which is what an object with spin $Q^{2} / 2$ collects. The exchange statistics for two quasiparticles of charge $Q$ each should then be $\theta / \pi=Q^{2}$.

Numerics. - We compare the predictions that the charge varies continuously as a function of the scaling variable $\mu_{s}(\infty) / \Delta(\infty)$ according to Eq. (5) in the absence of the axial vortex $\left[a_{5}(\infty)=0\right]$ and that the charge rerationalizes to $Q=1 / 2$ in the presence of the axial half-vortex $\left[a_{5}(\infty)=1 / 2\right]$ to results from an exact diagonalization in a lattice model. The model we consider is the $\pi$-flux phase [15] for electrons hopping on a square lattice, to which we add a dimerization pattern of the hopping amplitudes that realizes, on the lattice, the mass and axial vortices of Refs. [3,4]. We then study the charge bound to these singularities as a function of the staggered chemical potential $\mu_{s}$ for the cases $a_{5}(\infty)=0,1 / 2$ (see also [16]).

Consider a square lattice as in Fig. 2 on which spinless fermions hop. The square lattice can be divided into two interpenetrating sublattices $A$ (open circles in Fig. 2) and $B$ (filled circles in Fig. 2), such that all nearest neighbors of sites in $A$ belong to $B$, and vice versa. Here we construct the tight-binding Hamiltonian as $H=-\sum_{r \in A} \sum_{j=1}^{4}\left(t_{r, j}^{\pi}+\right.$ $\left.\delta t_{r, j}\right) a_{r}^{\dagger} b_{r+s_{j}}+$ H.c., where $r=\left(m_{1}, m_{2}\right) \in \mathbb{Z}^{2}$ labels the sites in $A$, located at positions $\left(m_{1}+m_{2}\right) \hat{x}+\left(m_{1}-m_{2}\right) \hat{y}$ in the square lattice. The $s_{j}$ are the four vectors (labeled counterclockwise, starting from the $+\hat{x}$ direction, by $j=$ $1,2,3,4)$ connecting a site in $A$ to one of its four nearestneighbor sites in $B$.

The kinetic energy in Eq. (1) follows from linearizing at half-filling the tight-binding dispersion when the only nonvanishing hopping amplitudes in units of $t>0$ are $t_{r, j}^{\pi}=$ $(-1)^{\left(m_{1}+m_{2}\right)\left(\delta_{j, 1}+\delta_{j, 3}\right)} t$. These hoppings define the $\pi$-flux phase (they are gauge equivalent to the case of uniform hoppings $t$ but with a magnetic flux of $\pi$ in units of $h / e$ threading each elementary plaquette, which is indicated in Fig. 2).

A staggered chemical potential $+\mu_{s}$ on sublattice $A$ and $-\mu_{s}$ on sublattice $B$ induces the bilinear $\bar{\Psi} M_{3} \Psi$ in Eq. (1) [9]. A dimerization, such as shown in Fig. 2(a), arises from $\delta t_{r, j} \propto(-1)^{m_{1}+m_{2}}\left(\delta_{j, 1}-\delta_{j, 3}\right) t_{r, j}^{\pi}$. Such dimerizations induce the mass bilinears $\bar{\Psi} M_{1,2} \Psi$ in Eq. (1) [3]. A dimerization, such as shown in Fig. 2(b), arises from $\delta t_{r, j} \propto\left(\delta_{j, 3}-\right.$ (a)

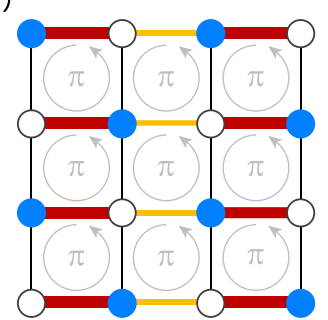

(c)

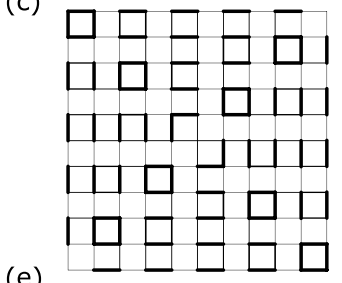

(e)

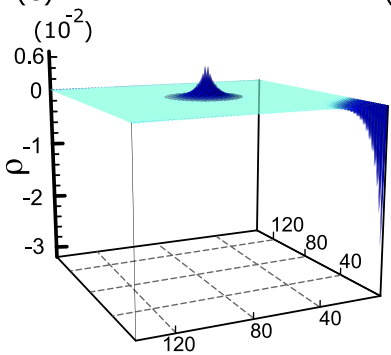

(b)

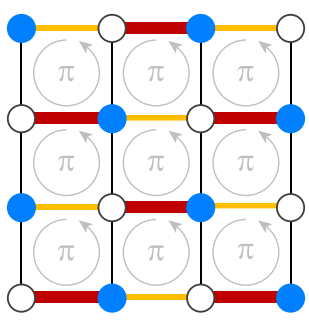

(d)

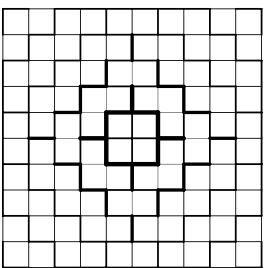

(f)

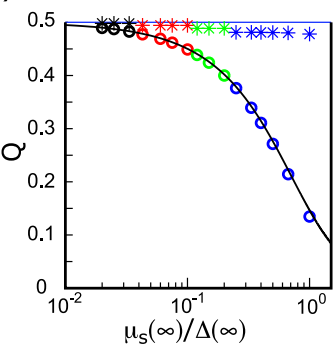

FIG. 2 (color online). (a) The columnar dimerization pattern, as indicated by the coloring (shading) of the bonds, for the nearest-neighbor hopping on a square lattice in the background of the $\pi$-flux phase that opens up the gap $\Delta(\infty)$. A staggered chemical potential, as indicated by the distinction between sites of sublattice $A$ and $B$ opens up the gap $\mu_{s}(\infty)$. (b) The staggered dimerization pattern for the nearest-neighbor hopping on a square lattice that generates an axial gauge field. (c) A charge1 vortex in the columnar dimerization (a). (d) A charge- $1 / 2$ vortex in the staggered dimerization (b) with core radius $c=0.3$. (e) The fermion density profile of (c) for a square lattice with open boundary conditions and $144 \times 144$ sites. (f) The fermion number as a function of the scaling variable $\mu_{s}(\infty) / \Delta(\infty)$ in the presence of the single charge 1 vortex (c) or with the addition of the axial charge $1 / 2$ vortex (d) with core radius $c=0.01$. The staggered chemical potential $\mu_{s}$ takes the values $0.01 t$ (black, points $1-3$ ), $0.03 t$ (red, points $4-7$ ), $0.06 t$ (green, points $8-10$ ), and $0.1 t$ (blue, points 11-15). The thick and thin lines are the prediction (5) without the axial vortex and $Q=1 / 2$, respectively.

$\left.\delta_{j, 1}\right) t_{r, j}^{\pi}$. Such dimerizations induce the axial bilinears $\bar{\Psi} \gamma_{1,2} \gamma_{5} \Psi$ in Eq. (1).

A charge- $n$ vortex in the columnar dimerization pattern is defined by

$$
\begin{gathered}
\delta t_{r, j}=t_{r, j}^{\pi} \frac{\Delta(\infty)}{2 t}\left(\cos n \theta \cos \phi_{j}-\sin n \theta \sin \phi_{j}\right), \\
\cos \theta=\frac{m_{1}+m_{2}}{|r|}, \quad|r|=\sqrt{2\left(m_{1}^{2}+m_{2}^{2}\right)}, \\
\phi_{j}=\pi\left(m_{1}+m_{2}\right)+j(\pi / 2),
\end{gathered}
$$


and shown in Fig. 2(c) when $n=1$. A half-vortex with core radius $c$ in the staggered dimerization pattern is defined by

$$
\delta t_{r, j}=\frac{t_{r, j}^{\pi}}{2|r|} \tanh \frac{|r|}{c}\left[\left(\delta_{j, 3}-\delta_{j, 1}\right) \cos \theta+\left(\delta_{j, 4}-\delta_{j, 2}\right) \sin \theta\right]
$$

and shown in Fig. 2(d).

The charge-density profile is obtained by adding the contributions from all exact single-particle eigenstates that are filled: negative energy states plus the bound state with energy within the gap that appears as a consequence of the vortex. The fermionic density profile of the vortex from Fig. 2(c) is shown in Fig. 2(e) for a lattice made of $144 \times 144$ sites with a weight concentrated either at the core of the vortex or on the boundary (open boundary conditions are used). The fermion number in the presence of a vortex is approximated by integrating the local fermion density in a disk surrounding the vortex that extends beyond the localization length of the induced bound state but remains insensitive to the fermion density that has accumulated at the boundary. Subtraction of the background fermion charge in the absence of the vortex is always implicit. The continuous dependence of the fermion number (5) on the scaling variable $\mu_{s}(\infty) / \Delta(\infty)$ is shown in Fig. 2(f) for the charge 1 vortex of Fig. 2(c). Also shown in Fig. 2(f) is the effect of the axial charge 1/2 vortex from Fig. 2(d) superimposed to the charge 1 vortex of Fig. 2(c); as anticipated we find that the fermion number stays close to $1 / 2$. We conclude that the agreement between the field theory and the numerics is good and improves as both $\mu_{s}(\infty) / \Delta(\infty) \rightarrow 0$ and $\Delta(\infty) / t \rightarrow 0$.

Energetics. - At the level of the field theory, the energy cost to separate vortices of opposite charge grows like the logarithm of their separation. Temperature fluctuations screen this interaction through the Kosterlitz-Thouless mechanism. Alternatively, it was shown in Ref. [4] that the bare interaction between vortices is screened at zero temperature by coupling them to an axial gauge field $A_{5 \mu}$ carrying a half-vortex. The issue of energetics on the lattice is more subtle than in the continuum approximation. The axial $U(1)$ symmetry of the field theory (1) is reduced to the finite group $\mathbb{Z}_{4}$ of rotations by $\pi / 2$ about a site of the square lattice when the dimerization pattern responsible for the gap at the Fermi energy is commensurate with the lattice. If so, the energy cost to create two vortices of opposite charge grows linearly with their separation. However, slight deformations of the hopping amplitudes away from the $\mathbb{Z}_{4}$-symmetric ones move the position of the Dirac points in the Brillouin zone, rendering the wave vector for the gap-opening dimerization pattern (which connects the two Dirac points) incommensurate with the lattice while preserving the bound state in the gap [3]. This incommensuration can restore an energy cost for the cre- ation of a vortex-antivortex pair with a logarithmic dependence on their separation, as is believed to happen in quantum dimer models, in the mechanism termed Cantor deconfinement in Ref. [17].

Summary. - We have calculated the irrational fermion number attached to vortices in a complex-valued Higgs field coupled to massive Dirac fermions in $(2+$ 1)-dimensional space-time. We also calculated the exchange statistics of these vortices and showed that it is irrational as soon as the fractional fermion number is. Deconfinement of the vortices at zero temperature requires the (axial gauge) coupling to a half-vortex [4]. Remarkably, this coupling rerationalizes the fermion number to the value $1 / 2$. We have compared our predictions for the induced fermion number with an exact diagonalization study of a planar tight-binding model with a static defective dimerization of the hopping amplitude and found a good agreement.

This work is supported in part by NSF Grant No. DMR0305482 (C. C. and C.-Y.H.), DOE Grant No. DE-FG0205ER41360 (R.J.), and DOE Grant No. DE-FG0291ER40676 (S.-Y.P.). A. S. thanks the Swiss NSF for its financial support. Use of the CNSI Computer Facilities at UCSB is gratefully acknowledged.

[1] R. Jackiw and C. Rebbi, Phys. Rev. D 13, 3398 (1976).

[2] W. P. Su, J. R. Schrieffer, and A. J. Heeger, Phys. Rev. Lett. 42, 1698 (1979).

[3] C.-Y. Hou, C. Chamon, and C. Mudry, Phys. Rev. Lett. 98, 186809 (2007).

[4] R. Jackiw and S.-Y. Pi, Phys. Rev. Lett. 98, 266402 (2007).

[5] J. Goldstone and F. Wilczek, Phys. Rev. Lett. 47, 986 (1981).

[6] M. J. Rice and E. J. Mele, Phys. Rev. Lett. 49, 1455 (1982).

[7] R. Jackiw and G. Semenoff, Phys. Rev. Lett. 50, 439 (1983).

[8] F. D. M. Haldane, Phys. Rev. Lett. 67, 937 (1991).

[9] G. W. Semenoff, Phys. Rev. Lett. 53, 2449 (1984).

[10] F. D. M. Haldane, Phys. Rev. Lett. 61, 2015 (1988).

[11] T. Jaroszewicz, Phys. Lett. B 146, 337 (1984).

[12] Y.-H. Chen and F. Wilczek, Int. J. Mod. Phys. B 3, 117 (1989).

[13] Z. Hlousek, D. Sénéchal, and S.-H. Tye, Phys. Rev. D 41, 3773 (1990).

[14] A. G. Abanov and P. B. Wiegmann, Nucl. Phys. B570, 685 (2000).

[15] For a review of the $\pi$-flux phase, see E. H. Lieb, Phys. Rev. Lett. 73, 2158 (1994).

[16] For a numerical study of the case $\mu_{s}=0$ and $\Phi_{5}=0$, see B. Seradjeh, C. Weeks, and M. Franz, Phys. Rev. B 77, 033104 (2008).

[17] Eduardo Fradkin, David A. Huse, R. Moessner, V. Oganesyan, and S. L. Sondhi, Phys. Rev. B 69, 224415 (2004); Ashvin Vishwanath, L. Balents, and T. Senthil, Phys. Rev. B 69, 224416 (2004). 